\title{
Aeromonas Hydrophilia as a Rare Cause of Septic Arthritis in a Hemodialysis Patient
}

\author{
Saarah Huurieyah Wan Rosli ${ }^{1}$, Chuan Hun Ding ${ }^{2}$, Asrul Abdul Wahab ${ }^{2}$
}

\begin{abstract}
:
Septic arthritis usually represents a direct invasion of joint space by various microorganisms, most commonly caused by bacteria. Most of the time, it is caused by Staphylococci spp. or Streptococci spp. This is a case of a 70-year-old Chinese man with underlying end stage renal failure on regular hemodialysis who presented with recurrent right shoulder pain and swelling. He was diagnosed with right shoulder septic arthritis whereby arthrotomy was performed. Intra-operative tissue specimen from his right shoulder grew Aeromonas hydrophilia which was susceptible to ceftriaxone, cefepime, ciprofloxacin, gentamicin and sulfamethoxazole-trimethoprim. He was given intravenous cefepime for 21 days and discharged after treatment completed.
\end{abstract}

Key words: Aeromonas hydrophila, septic arthritis

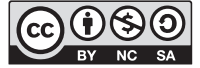

DOI: https://doi.org//0.3329/jom.v2 Ii2.502I 7

Copyright: (C) 2020 Rosli SHW et al. This is an open access article published under the Creative Commons Attribution-NonCommercial-NoDerivatives 4.0 International License, which permits use, distribution and reproduction in any medium, provided the original work is properly cited, is not changed in any way and it is not used for commercial purposes.

Received: 07 November 2020;

Accepted: 03 September 2020

\section{Introduction:}

Septic arthritis is a serious, life and limb threatening infection. If suspected, empirical treatment must be initiated immediately and must account for the most likely pathogens. Bacterial infection is responsible for most of the acute forms of septic arthritis and it is usually resulting in destructive form of arthritis. ${ }^{1}$ Staphylococcus aureus by large was reported as the main pathogen to be isolated in this condition. ${ }^{2}$ Aeromonas hydrophila is considered as an atypical bacterium causing acute septic arthritis. Isolation and identification of this bacteria as the caused of septic arthritis is very important as it implicates the choice of antibiotic to be used in treatment of this infection.

1. Medical Microbiologist Trainee, Faculty of Medicine and Health Sciences, Universiti Sains Islam Malaysia, Pandan Indah, 55100 Kuala Lumpur, Malaysia

2. Associate Professor, Department of Medical Microbiology and Immunology, Faculty of Medicine, Universiti Kebangsaan Malaysia, 56000 Cheras, Kuala Lumpur, Malaysia

Corresponding author: Associate Professor Dr. Asrul Abdul Wahab, Department of Medical Microbiology and Immunology, Universiti Kebangsaan Malaysia, 56000 Cheras, Kuala Lumpur, Malaysia, Email: asrulwahab@hotmail.com Phone: +603-9145 9530

\section{Case summary:}

A 70-year-old Chinese man with underlying end stage renal failure on regular hemodialysis presented with three days history of right shoulder pain and swelling. This was associated with movement limitation on the shoulder. He had no fever. He denied history of trauma to the shoulder. He had similar presentation about a month prior, whereby diagnosis of acute septic arthritis was made. He underwent arthrotomy washout previously but synovial fluid for microbiological culture showed no bacterial growth. Physical examination revealed presence of swelling at the anterior part of the right shoulder joint. The skin overlying the swollen join was warm. The joint was tender on palpation with very limited range of movement. Otherwise no other significant findings noted. Baseline blood investigations showed presence of leukocytosis predominantly neutrophils with the count of $17.3 \times 10^{9} / \mathrm{L}$ and $15.7 \times 10^{9} / \mathrm{L}$ respectively. The Creactive protein was also raised at $30.38 \mathrm{mg} / \mathrm{dL}$. The results were as shown in Table 1. The right shoulder plain radiograph showed right shoulder degenerative changes and the ultrasound of the same joint indicated the presence of infected complex bursitis, but it was unable to rule out underlying septic arthritis. Thus, the diagnosis of acute septic arthritis was made clinically. He was started on empirical intravenous cefuroxime $750 \mathrm{mg}$ eight hourly. 
Table I: The different of the laboratory parameters on the day of admission and on the day of discharged.

\begin{tabular}{lccc}
\hline Parameters & On the day of admission & On the day of discharged & Normal range \\
\hline White cell count & $17.3 \times 10^{9} / \mathrm{L}$ & $9.2 \times 10^{9} / \mathrm{L}$ & $(4.1-11.4) \times 10^{9} / \mathrm{L}$ \\
Neutrophils & $15.7 \times 10^{9} / \mathrm{L}$ & $7.1 \times 10^{9} / \mathrm{L}$ & $(3.9-7.1) \times 10^{9} / \mathrm{L}$ \\
& $(90.6 \%)$ & $(77.1 \%)$ & \\
Hemoglobin (g/dL) & 10.7 & 9.8 & $11.8-16.9$ \\
C-reactive protein (mg/dL) & 30.38 & 6.28 & $<0.05$ \\
\hline
\end{tabular}

Incisional drainage of the right shoulder together with arthrotomy washout were performed. Intraoperative findings include cloudy synovial fluid, slough tissue, unhealthy synovial tissue, chondral erosion over lateral humeral head. Tissue specimen was taken and send for microbiology culture. Blood sample was also taken for similar purpose. Tissue specimen grew colonies with hemolysis on blood agar and non-lactose fermenting coliforms on MacConkey agar (Figure 1A and 1B).


Fig.-1A: demonstrated hemolytic colonies produced by these bacteria on the sheep blood agar and Fig.-1B: showed nonlactose fermenting bacteria isolated on the MacConkey agar.

Gram stain reaction showed the presence of gram-negative bacteria. Oxidase test was positive. The bacteria were motile in semisolid agar and producing acid-alkaline reaction in triple sugar iron medium (Figure 2A and 2B).

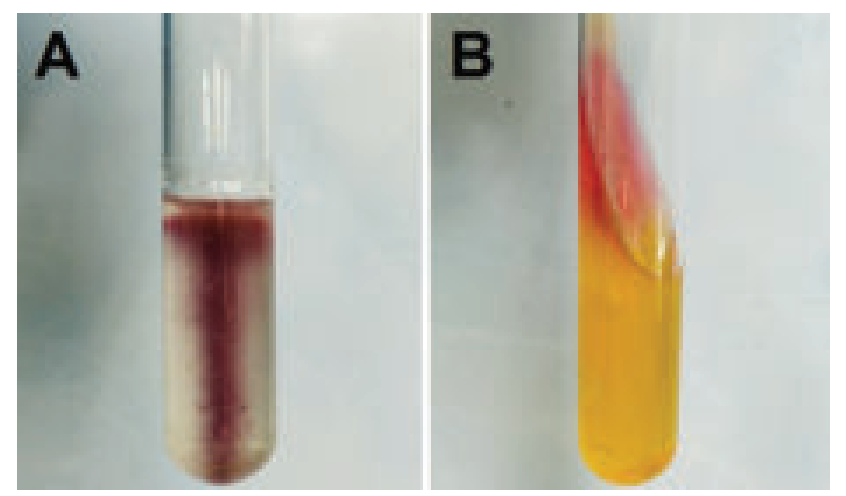

Fig.-2A: demonstrated that the bacteria were motile in the semisolid agar and Fig.-2B: showed it produced acidalkaline reaction in triple sugar iron medium.
Further laboratory test by API 20NE (Biomériuex, France) bacterial identification kits showed Aeromonas hydrophilia with $99.9 \%$ identification (Table 2). The organism is susceptible to ciprofloxacin, gentamicin, ceftriaxone, cefepime and sulfamethoxazole-trimethoprim. The blood for culture was negative. Thus, the antibiotic was changed to intravenous cefepime. The treatment was completed for 21 days. Subsequently, he was discharged well.

Table - II

The biochemical reaction of the bacteria by the API20NE identification kit. The bacteria was identified as Aeromonas hydrophila based on this profile.

\begin{tabular}{lll}
\hline Tests & Active Ingredients & Results \\
\hline $\mathrm{NO}_{3}$ & Potassium nitrate & Positive \\
$\mathrm{TRP}$ & L-tryptophane & Positive \\
$\mathrm{GLU}$ & D-glucose & Positive \\
$\mathrm{ADH}$ & L-arginine & Positive \\
URE & Urea & Negative \\
ESC & Esculin & Positive \\
GEL & Gelatin & Positive \\
PNPG & 4-nitropheny-bD-galactopyranoside & Positive \\
GLU & D-glucose (assimilation) & Positive \\
ARA & L-arabinose & Positive \\
MNE & D-mannose & Positive \\
MAN & D-mannitol & Positive \\
NAG & N-acetyl-glucosamine & Positive \\
MAL & D-maltose & Positive \\
GNT & Potassium gluconate & Positive \\
CAP & Capric acid & Positive \\
ADI & Adipic acid & Negative \\
MLT & Malic acid & Positive \\
CIT & Trisodium citrate & Negative \\
PAC & Phenylacetic acid & Negative \\
OX & Oxidase & Positive \\
\hline & &
\end{tabular}




\section{Discussion:}

Staphylococcus spp. and Streptococcus spp. were reported as the main causative agent of septic arthritis. ${ }^{3}$ Similarly, as reported in the literature, septic arthritis among hemodialysis patients was mainly caused by Staphylococcus aureus. ${ }^{4}$ Aeromonas hydrophila is a rare pathogen causing septic arthritis in human. It contributed to only $0.77 \%$ of all cases of septic arthritis. ${ }^{5}$ Prior wound around joint area which subsequently exposed to contaminated watery environment perhaps occurred in most reported cases. ${ }^{5-7}$ There was also a reported case of catheter related sepsis in a patient with chronic kidney disease on regular dialysis caused by Aeromonas hydrophila. ${ }^{8}$ Similar to other acute septic arthritis, patients will present with joint pain and limited movement over the affected joint. Fever was also one of the most common clinical manifestations and it was noted in $90 \%$ of the patients. ${ }^{9}$ In term of basic laboratory investigations, leukocytosis and increased C-reactive protein level were among the common findings. 5,6

Aeromonas hydrophila are gram-negative, non- spore forming facultative anaerobic rods bacteria which usually produced beta hemolytic colonies on blood agar. They ferment carbohydrates with acid and gas production. The organisms grow well on MacConkey agar producing nonlactose fermenter colonies. They have cytochrome oxidase, and this feature distinguishes them from Enterobacteriaceae spp. Identification of Aeromonas to the genus level is generally not difficult, but misidentifications may occur with different simplified biochemical identification kits. ${ }^{10}$ Identification can be done by different systems, among them API system. Both API 20E and API20NE identification kits can be used in identification of these bacteria with some limitations mainly due to limited database of Aeromonas spp. in both kits. API20NE that was used in this case showed 99.9\% confidence in identification of Aeromonas hydrophila. Aeromonas sobria is another Aeromonas spp. that is able to be identified by API20NE identification kit. The ability to hydrolyzed esculin is an important distinguishing property differentiates between these two genera. Aeromonas hydrophila is able to hydrolyze esculin but not Aeromonas sobria. ${ }^{11}$ Molecular identification of Aeromonas species is complex whereby previous study had demonstrated the need for using more than one method for correct identification. In that study, molecular methods targeting the GCAT, $16 \mathrm{~S}$ rDNA and $r p o D$ genes were used for identification of Aeromonas species. ${ }^{12}$ Perhaps, molecular identification can be sought out should the biochemical reaction is not able to determine the genus confidently.

As described in most clinical cases of Aeromonas hydrophila septic arthritis, the clinical management would require both medical and surgical intervention. The intravenous antibiotic depending on the susceptibility pattern was used initially before it was changed to oral antibiotic. Usually, prolonged antibiotic used is required. Arthrotomy and joint washout were performed in most of the previous cases to treat the infected joint. The clinically important Aeromonas species are regularly resistant to penicillin, ampicillin, cefazolin and ticarcillin. ${ }^{13}$ Most were susceptible to third or fourthgeneration cephalosporins, aminoglycosides, ciprofloxacin, and imipenem. Therefore, third- or fourth-generation cephalosporins as well as fluoroquinolones should be considered the antibiotic treatments of choice. Fluoroquinolones appear to be very active against Aeromonas species. Unfortunately, there are chromosomal mutations and plasmid-mediated quinolone resistance in environmental Aeromonas spp. strains. ${ }^{14}$

\section{Conclusion:}

In summary, this is a case of relatively common clinical problem, septic arthritis of the shoulder joint, caused by an unusual organism, Aeromonas hydrophilia. It requires surgical debridement with antibiotics. Third-generation cephalosporins and quinolones are considered the antibiotics of choice. Clinician should be aware that their patient population may be at increased risk for developing skin and joint infections by these atypical organisms particularly those with history of prior wound that is exposed to watery environment.

\section{Acknowledgement:}

Authors would like to thank the Dean of the Faculty of Medicine, Universiti Kebangsaan Malaysia for his support to publish this article.

\section{Conflict of interest: None}

\section{References:}

1. Nair R, Schweizer ML, Singh N. Septic Arthritis and Prosthetic Joint Infections in Older Adults. Infectious Disease Clinical North Am. 2017; 31:715.

2. Dubost JJ, Soubrier M, De Champs C, Ristori JM, Bussiére JL, Sauvezie B. No changes in the distribution of organisms responsible for septic arthritis over a 20 year period. Ann Rheum Dis. 2002; 61(3): 267-9.

3. Gupta MN, Sturrock RD, Field M. A prospective 2-year study of 75 patients with adult-onset septic arthritis. Rheumatology(Oxford). 2001; 40(1): 24-30.

4. Al-Nammari SS, Gulati V, Patel R, Bejjanki N, Wright M. Septic arthritis in haemodialysis patients: a seven-year multicentre review. J Orthop Surg (Hong Kong). 2008; 16(1): 54-7. 
5. Tseng CC, Wu CD, Cheng SC, Lin WT, Chan HT. Septic arthritis caused by Aeromonas hydrophila. Formosan Journal of Musculoskeletal Disorders. 2013; 4: 53-55.

6. Elwitigala JP, Higgs DS, Namnyak S, White JW, Yaneza A. Septic arthritis due to Aeromonas hydrophila: case report and review of the literature.Int J Clin Pract Suppl. 2005 ;147:121-4.

7. Danaher PJ, Mueller WP. Aeromonas hydrophila septic arthritis. Mil Med. 2011; 176 (12): 1444-6.

8. Muhammad Abdul Mabood Khalil, Abdur Rehman, Waqar Uddin Kashif, Manickam Rangasami, and Jackson Tan, A Rare Case of Aeromonas Hydrophila Catheter Related Sepsis in a Patient with Chronic Kidney Disease Receiving Steroids and Dialysis: A Case Report and Review of Aeromonas Infections in Chronic Kidney Disease Patients. Case Reports in Nephrology. 2013, Article ID 735194, doi:10.1155/2013/ 735194.

9. Lim HK, Bair MJ, Lee CM, Liu CP, Tseng HK, Wang JM. Aeromonas hydrophila infection: 6-year experience and literature review. J Taiwan Emerg Med. 2010; 12: 1-7.
10. Awan MB, Ahmed MM, Bari A, Saad AM. Biochemical characterization of the aeromonas species isolated from food and environment. Pak J Physiol. 2005; 1(1-2).

11. Abbott SL, Cheung WK, Janda JM. The genus Aeromonas: biochemical characteristics, atypical reactions, and phenotypic identification schemes. J Clin Microbiol. 2003; 41(6): 2348-57.

12. Puthucheary SD, Puah SM, Chua KH. Molecular characterization of clinical isolates of Aeromonas species from Malaysia. PLoS One. 2012; 7(2): e30205. doi: 10.1371/ journal.pone.0030205.

13. Janda JM, Abbott SL. 2010 The genus Aeromonas: taxonomy, pathogenicity, and infection. Clinical Microbiol Review. 23:35-73.

14. Han JE, Kim JH, Cheresca CH, et al. 2012 First description of the qnrS-like (qnrS5) gene and analysis of quinolone resistance-determining regions in motile Aeromonas spp. from diseased fish and water. Res Microbiol. 163:73-79. 